\title{
Evaluación de la aproximación al aprendizaje de estudiantes de ciencias de la vida usando la versión revisada del Study Process Questionnaire (R-SPQ-2F)
}

Leiva-Brondo, M. a, Cebolla-Cornejo, J. b Andrés-Colás, N. c, Peiró, R d, Pérez-de Castro, A. ${ }^{\mathrm{e}}$

amileibro@btc.upv.es, Departamento de Biotecnología bjaicecor@btc.upv.es, Departamento de Biotecnología, ㄷnuanco@btc.upv.es, Departamento de Biotecnología, dropeibar@btc.upv.es, Departamento de Biotecnología, eanpede1@btc.upv.es, Departamento de Biotecnología, Universitat Politècnica de València.

\begin{abstract}
The revised version of Study Process Questionnaire (R-SPQ-2F) analyzes the approach to learning on different scales. In the present study we analyzed the answers to the questionnaire of students of degree and master level of different subjects related to the sciences of the life in the sociocultural context of Spain. The adjustment of the model analyzed by the alpha of Cronbach was adequate to measure the approach to learning, deep or superficial, but not for the subscales of measurement of strategy and motivation. The students mainly expressed a deep learning approach. Although differences were detected among students according to subjects, they were not observed at the global level for the level of studies (degree or master), year or gender.
\end{abstract}

Keywords: Deep and surface student learning approach, motivation, biotechnology, plant breeding.

\footnotetext{
Resumen

El cuestionario revisado Study Process Questionnaire ( $R-S P Q-2 F)$ analiza la aproximación al aprendizaje en diferentes escalas. En el presente estudio se analizaron las respuestas al cuestionario de alumnos de grado y máster de distintas asignaturas relacionadas con las ciencias de la vida en el entorno sociocultural de España. El ajuste del modelo analizado mediante el alfa de Cronbach fue adecuado para medir la aproximación al aprendizaje, profunda o superficial, pero no para las subescalas de medición de estrategia y motivación. Los alumnos manifiestaron mayoritariamente una aproximación profunda al aprendizaje. Si bien se detectaron diferencias entre los estudiantes según asignaturas, no se observaron a nivel global para el nivel de estudios (grado o máster), curso o género.
}

Palabras clave: aproximación al aprendizaje profundo y superficial, motivación, biotecnología, mejora genética vegetal. 


\section{Introducción}

La educación universitaria no es obligatoria en la mayoría de los países, al contrario que los estudios de etapas previas como secundaria o primaria. Los alumnos son voluntarios y tienen diferentes opciones una vez acabada su educación obligatoria, como es el mercado laboral, la formación profesional o la formación especializada de otro tipo. Aunque es cierto que puede haber influencias personales, familiares o sociales para iniciar los estudios universitarios, la motivación principal suele ser intrínseca. Una encuesta reciente realizada entre más de 60.000 estudiantes europeos (QS Enrolment Solutions, 2017) muestra que sólo un porcentaje menor al $25 \%$ de los estudiantes eligen cursar estudios superiores por motivos externos a ellos. Es cierto también que la elección del título universitario en concreto tiene limitaciones externas, tales como la nota de acceso o restricciones económicas, como el lugar de impartición de los estudios o el coste de la matrícula (principalmente en universidades privadas o si no hay ayudas públicas disponibles). Sin embargo, las asignaturas que se cursan una vez elegidos los estudios universitarios son obligatorias, a excepción de las asignaturas optativas, que suelen estar en cursos superiores. Por tanto, la motivación e implicación en cada asignatura puede variar en función de factores intrínsecos y extrínsecos del estudiante. Esta motivación afecta al aprendizaje del alumno, pudiendo ser este superficial o profundo (Biggs, Kember \& Leung, 2001).

Los factores que afectan al tipo de aprendizaje del alumno en cada asignatura son múltiples; algunos de ellos dependen del profesor y de cómo enfoca la enseñanza de la asignatura (Biggs, Kember \& Leung, 2001). Por ello, es clave para conseguir maximizar el aprendizaje de los alumnos localizar aquellos factores modificables sobre los que se pueda actuar.

La aproximación del estudiante al aprendizaje (student approaches to learning) fue desarrollada inicialmente por Marton and Säljö (Marton \& Säljö, 1976b, 1976a) y llevó al desarrollo de la teoría del constructivismo. Esta teoría supone que el conocimiento debe ser construido dentro de la estructura cognitiva personal de cada individuo, y es fundamentalmente personal, pero depende de las experiencias del entorno de aprendizaje y de las interacciones sociales (Grabinger \& Dunlap, 1995). Los estudiantes más exitosos son los que participan metacognitivamente, motivados y con comportamiento activo en su proceso de aprendizaje (Zimmerman, 1986). Aunque el proceso por el cual los estudiantes se aproximan al aprendizaje es complejo, depende de factores contextuales y personales (Schmeck, Ribich \& Ramanaiah, 1977; Biggs, 1978). La aproximación al aprendizaje del alumno se puede clasificar de diversas maneras (Frăsineanu, 2013), pero los dos extremos serían el aprendizaje superficial y el aprendizaje profundo (Marton \& Säljö, 1976b; Graham, Entwistle \& Ramsden, 1984; Gijbels et al., 2005). El aprendizaje superficial se caracteriza por una falta de conexión personal, el uso de la memoria y la falta de reflexión, mientras que el aprendizaje profundo implica conexiones personales, reflexión sobre el significado y compresión (Frăsineanu, 2013). Los estudiantes que adoptan una aproximación superficial en una asignatura suelen estar más preocupados por la calificación y utilizan la memorización, mientras que los que utilizan una aproximación profunda persiguen el aprendizaje y comprender (Gijbels et al., 2005; Mogre \& Amalba, 2014). Se han utilizado aproximaciones cualitativas (Marton, 1981) o cuantitativas (Graham, Entwistle \& Ramsden, 
1984; Biggs, 1987) para medir la aproximación al aprendizaje. Todas ellas mantienen la dicotomía superficial-profundo (Prosser \& Trigwell, 1999; Gijbels et al., 2005), si bien se pueden identificar otras aproximaciones estratégicas o motivacionales (Entwistle, 1991; Biggs, Kember \& Leung, 2001; Gijbels et al., 2005). El estudio de la aproximación que utiliza el alumno para el aprendizaje se ha utilizado para analizar diferencias de género (Wilson, Smart \& Watson, 1996), efectividad de intervenciones (Gordon \& Debus, 2002), relación con objetivos de aprendizaje (Boyle, Duffy \& Dunleavy, 2003) o estabilidad del aprendizaje a lo largo del tiempo (Fox, McManus \& Winder, 2001). Por ejemplo, se ha visto que una aproximación profunda suele estar asociada con una mejor calidad del aprendizaje (Zeegers, 2001; Snelgrove \& Slater, 2003; Salamonson et al., 2013). También se ha visto que hay relación entre la taxonomía Structure of the Observed Learning Outcome, SOLO, y el aprendizaje profundo (Rossum \& Schenk, 1984; Crawford et al., 1998; Trigwell, Prosser \& Waterhouse, 1999). Esta relación puede usarse para mejorar el aprendizaje y el logro de los objetivos académicos, modificando, por ejemplo, la carga de trabajo o los procedimientos de evaluación (Zeegers, 2001).

Hay multitud de instrumentos disponibles para evaluar la aproximación al aprendizaje de los estudiantes, tales como Study Attitudes and Methods Revised Short Form (SAMS Short Form) (Michael, Michael \& Zimmerman, 1985), Revised Approaches to Studying Inventory (Entwistle \& Tait, 1995), Inventory of Learning Process-Revised (ILP-R) (Schmeck, Ribich \& Ramanaiah, 1977), Learning and Study Strategies Inventory (LASSI) (Weinstein, 1987) o Inventory of Learning Styles (Vermunt, 1994), entre otras. Uno de los más utilizados es el Study Process Questionnair (SPQ) (Biggs, 1987), que posteriormente fue revisado (R-SPQ2F) (Biggs, Kember \& Leung, 2001), y cuya validez ha sido contrastada por distintos estudios (Justicia et al., 2008; Immekus \& Imbrie, 2010; Socha \& Sigler, 2014).

El cuestionario de Biggs (Biggs, Kember \& Leung, 2001) se ha utilizado para establecer la relación entre la aproximación al aprendizaje y la personalidad, la adquisición de conocimiento, el rendimiento académico, la preferencia de estilo de aprendizaje, la autoeficiencia, la orientación de objetivos o las estrategias de autorregulación (Skogsberg \& Clump, 2003; Chamorro-Premuzic, Furnham \& Lewis, 2007; Phan, 2008; Furnham, Monsen \& Ahmetoglu, 2009).

El cuestionario SPQ original tenía 42 preguntas y tres aproximaciones al aprendizaje: superficial, profundo y de logro. El cuestionario R-SPQ-2F reduce considerablemente el número de ítems, lo cual falicita su uso. Consiste en 20 preguntas tipo Likert, con una escala de 5 puntos. Las respuestas de los alumnos a este cuestionario tienen en cuenta la aproximación al aprendizaje y las características individuales del estudiante (Socha \& Sigler, 2014). Este cuestionario no pretende clasificar a los estudiantes como aprendices superficiales o profundos, sino que trata de estimar el comportamiento del alumno en el contexto de aprendizaje concreto donde se realiza el cuestionario (Biggs, Kember \& Leung, 2001), en el que el estudiante opta por una aproximación al aprendizaje en función de factores intrínsecos y extrínsecos (Biggs, Kember \& Leung, 2001). Estos factores interaccionan entre sí y se adaptan a cada contexto de aprendizaje. El profesor tiene que proporcionar las actividades de aprendizaje adecuadas para que el alumno alcance los objetivos planteados, 
haciendo énfasis en lo que el estudiante hace (Shuell, 1986). Estudiantes que normalmente optan por un aprendizaje superficial pueden optar a un aprendizaje profundo si el contexto es el adecuado y viceversa (Biggs, Kember \& Leung, 2001). Los estudiantes son capaces de crear sus entornos de aprendizaje para optimizar su rendimiento, pero a menudo necesitan de ayuda para elegir las estrategias a emplear. Sin guía e instrucción su aprendizaje distará de ser óptimo (Socha \& Sigler, 2014).

El propósito principal de un buen profesor es fomentar que los estudiantes adopten un aprendizaje profundo y desincentivar el aprendizaje superficial (Biggs, 1999), por lo que medir la aproximación al aprendizaje del estudiante proporciona un índice de la calidad de la enseñanza (Biggs, Kember \& Leung, 2001). Por ejemplo, la utilidad de un resultado de aprendizaje puede fomentar el aprendizaje profundo, mientras que el tiempo de dedicación puede fomentar un aprendizaje superficial (Berger \& Karabenick, 2011). El cuestionario RSPQ-2F puede indicar el contexto previo, cómo se enfoca una determinada tarea o cómo afecta el contexto de aprendizaje a los alumnos (Biggs, Kember \& Leung, 2001).

Factores del estudiante que pueden influir en la aproximación del estudiante al aprendizaje son (Gijbels et al., 2005):

- La edad. A mayor edad normalmente se produce un mayor aprendizaje profundo (Gow \& Kember, 1990; Richardson \& King, 1998; Gijbels et al., 2005), posiblemente debido a que se tiene más motivación intríseca (Harper \& Kember, 1986), lo cual puede explicar su mayor éxito (Zeegers, 2001).

- Género. En general las mujeres tienden a un mayor aprendizaje profundo que los hombres (Severiens \& Dam, 1998), aunque algunos estudios no detectan diferencias (Zeegers, 2001; May et al., 2012).

- Diferencias culturales. En función del país o cultura de origen se han visto diferencias a la hora de aproximarse al aprendizaje (Biggs, Kember \& Leung, 2001; Justicia et al., 2008; Leung, Ginns \& Kember, 2008; Immekus \& Imbrie, 2010; Salamonson et al., 2013).

- Carga de trabajo. Una mayor carga de trabajo se ve asociada normalmente a una aproximación superficial (Entwistle \& Tait, 1990; Gow \& Kember, 1990; Leung, Mok \& Wong, 2008).

Otros factores que afectan a la aproximación al aprendizaje pueden ser el tipo de estudios, las actividades en el aula, el sistema de evaluación, el entono institucional o el curso en el que se enmarca la asignatura (Zeegers, 2001). Por ejemplo, se ha visto que el aprendizaje profundo es menor en cursos superiores que en curso iniciales (Biggs, 1987; Zeegers, 2001), lo cual puede indicar que el entorno de enseñanza-aprendizaje no requiere un aprendizaje profundo o que el estudiante no lo percibe así (Zeegers, 2001), de forma que una aproximación superficial puede ser suficiente para lograr los objetivos del curso.

Las aproximaciones profundas y superficiales pueden subdividirse entre estrategias y motivos. Las estrategias son las actividades, métodos o procesos que el estudiante utiliza durante su aprendizaje, mientras que los motivos son las razones y los objetivos que le llevan 
a esos comportamientos (Justicia et al., 2008). Aunque estas asociaciones no se aprecian en todos los estudios (Justicia et al., 2008; Immekus \& Imbrie, 2010; Socha \& Sigler, 2014).

Existen diferentes estudios donde se utiliza el cuestionario de Biggs para evaluar la aproximación al aprendizaje de los estudiantes y se ha evaluado su eficacia en distintos contextos socioculturales. Por ejemplo, en estudios de Medicina en Ghana los estudiantes mostraron mayoritariamente una aproximación al aprendizaje profundo (Mogre \& Amalba, 2014) y resultados similares se obtuvieron en Arabia Saudí (Shaik et al., 2017). En este último caso se relacionó el aprendizaje profundo con un mayor número de horas de estudio y mejores calificaciones. Por otro lado, estudiantes de Derecho en Holanda (Gijbels et al., 2005) mostraron una mayor puntuación en aprendizaje profundo que superficial, aunque muchos obtuvieron puntuaciones bajas en ambas aproximaciones al aprendizaje. En este mismo estudio, no se obtuvieron correlaciones significativas entre la evaluación y la resolución de problemas, pero sí se encontraron entre la edad y el género de los estudiantes (Gijbels et al., 2005). En otro estudio realizado en Australia con estudiantes de Química utilizando el cuestionario original de Biggs se observó que el aprendizaje profundo estaba relacionado con la evaluación de los objetivos de aprendizaje y que la edad era el mayor factor que influenciaba en la aproximación al aprendizaje y al rendimiento (Zeegers, 2001). Por otro lado, estudios realizados en Hong-Kong con el cuestionario no revisado de Biggs y entrevistas mostraron que el aprendizaje profundo está ligado positivamente a la edad y negativamente al curso en el que está el alumno (Gow \& Kember, 1990). Estudios realizados en Japón (Fryer et al., 2012) validaron el cuestionario de Biggs en relación con las aproximaciones profunda y superficial, pero no con las subescalas del cuestionario y muestran distintas relaciones que no aparecen en otros estudios y que podrían ser debidas a motivos culturales. Estudios realizados en China (Leung, Ginns \& Kember, 2008) mostraron que los estudiantes utilizaban aproximaciones más intermedias al aprendizaje con la memorización como una herramienta para lograr la compresión.

También se han realizado otros estudios comparando distintos modelos de elección de las preguntas que determinan el aprendizaje profundo o superficial a partir del cuestionario de Biggs (Socha \& Sigler, 2014). Uno de estos estudios comparó los resultados del trabajo de Biggs (Biggs, Kember \& Leung, 2001) realizados en Hong Kong, con estudios donde se evaluó el cuestionario en distintos entornos (España (Justicia et al., 2008), Estados Unidos (Immekus \& Imbrie, 2010; Socha \& Sigler, 2014) o Países Bajos (Stes, De Maeyer \& Van Petegem, 2013)). En el mencionado estudio también se indicó que puede haber diferencias culturales que expliquen diferentes resultados y mostró controversia respecto a la agrupación de las preguntas del test (Justicia et al., 2008; Socha \& Sigler, 2014). Un estudio que comparó los resultados de estudiantes de Hong Kong y Sidney evidenció diferencias que podían atribuirse a causas culturales (Leung, Ginns \& Kember, 2008).

Estos estudios han analizado la aproximación al aprendizaje en numerosos contextos, sin embargo no se dispone de un estudio pormenorizado que incluya las condiciones socioculturales de España en un entorno de aprendizaje de las ciencias de la vida. Estos resultados permitirían organizar las metodologías de enseñanza y fomentar el desarrollo de las competencias trasversales y específicas de cada titulación. 


\section{Objetivos}

En el presente estudio se pretende analizar las respuestas al cuestionario R-SPQ-2F de alumnos procedentes de distintas asignaturas, niveles y elección en un entorno de aprendizaje de las ciencias de la vida en España, así como evaluar la fiablidad del cuestionario en nuestras condiciones y hallar las relaciones entre las distintas escalas del cuestionario.

\section{Desarrollo de la innovación}

Los alumnos que respondieron al cuestionario R-SPQ-2F pertenecían a cinco asignaturas (tabla 1): Tres de ellas del Grado en Biotecnología: Genética General (obligatoria de primer curso), Aspectos legales y sociológicos de la biotecnolgía (obligatoria de cuarto curso) y Experimentación en Biotecnología (optativa de cuarto curso). Las otras dos asignaturas pertenecen al máster en Mejora Genética Vegetal: Recursos Fitogenéticos y Mejora genética del rendimiento en los sistemas agrarios. Los alumnos cursaron las asignaturas en el curso 2018-19, con la excepción de la asignatura Mejora genética del rendimiento en los sistemas agrarios, para la cual se incluyeron además datos del curso 2017-18.

Tabla 1. Asignaturas implicadas en el estudio

\begin{tabular}{ccccccc}
\hline Asigantura & Nivel & Curso & Tipo & Idioma & Créditos & $\mathbf{N}^{\mathbf{0}}$ alumnos \\
\hline GG & Grado & 1 & Obligatoria & Castellano/ Inglés & 6,0 & 127 \\
ASLB & Grado & 4 & Obligatoria & Castellano & 6,0 & 99 \\
EB & Grado & 4 & Optativa & Castellano & 4,5 & 53 \\
RF & Máster & 1 & Obligatoria & Castellano & 5,0 & 19 \\
MG & Máster & 2 & Obligatoria & Castellano & 5,0 & 13 y 17 \\
\hline
\end{tabular}

GG: Genética General; ASLB: Aspectos sociológicos y legales de la biotecnología; EB: Experimentación en biotecnología; RF: Recursos Fitogenéticos; MG: Mejora genética del rendimiento en los sistemas agrarios.

El cuestionario R-SPQ-2F (Biggs, Kember \& Leung, 2001) se proporciona a los alumnos a través de la plataforma en línea de aprendizaje PoliformaT basada en Sakai. Este cuestionario incluye 20 preguntas (tabla 2) divididas en dos escalas de aproximación profunda y superficial. La escala de aproximación profunda (DA) incluye subescalas de motivación profunda (DM) y estrategia profunda (DS); a su vez, la aproximación superficial (SA) incluye subescalas de motivo superficial (SM) y estrategia superficial (SS). Cada subescala consta de cinco elementos en una escala de tipo Likert de 5 puntos que va desde 1 ("Nunca o muy raras veces") a 5 ("Siempre"). Para obtener las puntuaciones de las escalas principales para la DA, se utilizaron los resultados de las preguntas $1,2,5,6,9,10,13,14,17$ y 18 , y para SA las preguntas $3,4,7,8,11,12,15,16,19$ y 20 . Para las subescalas se utilizaron las siguientes preguntas: 1, 5, 9, 13 y 17 para DM; 2, 6, 10, 14 y 18 para DS; 3, 7, 11, 15 y 19 para SM; y 4, 8, 12, 16 y 20 para SS. También se calculó la diferencia entre la escala DA y la escala SA. El cuestionario utilizado fue una traducción al español. (Muñoz San Roque, Prieto Navarro \& Torre Puente, 2012) para grupos con castellano como medio de instrucción 
y la versión inglesa se utilizó para grupos con el inglés como medio de instrucción. Los resultados se analizaron utilizando Statgraphics centurión XVII (Statpoint Technologies, Inc.). Se calcularon las correlaciones entre los factores y los valores alfa de Cronbach.

Tabla 2. Preguntas del cuestionario R-SPQ-2F traducidas a castellano (Muñoz San Roque, Prieto Navarro \& Torre Puente, 2012)

\section{Cuestiones}

1. Me doy cuenta de que estudiar me proporciona a veces un sentimiento de profunda satisfacción personal.

2. Al elaborar o estudiar un tema, no me encuentro satisfecho hasta que me he formado mis propias conclusiones sobre él.

3. Mi objetivo es aprobar el curso haciendo el mínimo trabajo posible.

4. Sólo estudio seriamente lo que se da en las clases o lo que está en los programas detallados de las asignaturas.

5. Me parece que cualquier tema puede llegar a ser altamente interesante una vez que te metes en él.

6. Encuentro interesantes la mayoría de los nuevos temas y empleo tiempo extra intentando obtener mayor información sobre ellos.

7. Cuando veo que la asignatura no me resulta muy interesante, trabajo lo menos posible.

8. Aprendo algunas cosas repitiéndolas hasta que me las sé de memoria incluso aunque no las comprenda.

9. Estudiar temas académicos puede ser a veces tan apasionante como leer una buena novela o ver una buena película.

10. Me hago preguntas a mí mismo sobre los temas importantes hasta que los comprendo totalmente.

11. Creo que puedo aprobar la mayoría de las evaluaciones memorizando los aspectos clave en lugar de intentar comprenderlos.

12. Generalmente limito mi estudio a lo que está específicamente ordenado, porque creo que es innecesario hacer cosas extra.

13. Trabajo duro en mis estudios porque encuentro los temas interesantes.

14. Empleo bastante de mi tiempo libre en buscar más información sobre temas interesantes que se han discutido en las diferentes clases.

15. Me parece que no ayuda estudiar los temas en profundidad. Confunde y hace perder el tiempo cuando todo lo que se necesita es un conocimiento por encima de los temas.

16. Creo que los profesores no deberían esperar que los alumnos dedicaran mucho tiempo a estudiar cosas que no van a caer en el examen.

17. Voy a la mayoría de las clases con preguntas a las que desearía encontrar respuesta.

18. Es muy importante para mí echar un vistazo a la mayoría de las lecturas recomendadas que tienen que ver con las clases.

19. No le encuentro sentido a aprender contenidos que probablemente no caerán en el examen.

20. Me parece que la mejor manera de aprobar es recordar las respuestas de las preguntas que con más probabilidad pueden caer en el examen. 


\section{Resultados}

El total de respuestas obtenidas fue de 182 , un $55 \%$ del total de alumnos posibles que podían responder, variando de $24,2 \%$ de Aspectos legales y sociológicos de la biotecnolgía a $86.7 \%$ de Mejora genética del rendimiento en los sistemas agrarios (tabla 3). La mayoría de estudiantes presentaron una aproximación profunda al aprendizaje en todas las asignaturas, niveles y género. Solamente 20 de los 182 alumnos que respondieron el cuestionario manifestaron un aproximación superficial mayor que la aproximación profunda. De estos 20 alumnos, el 50\% de ellos cursaban la asignatura Experimentación en biotecnología. Si se comparan los resultados del cuestionario entre asignaturas se observan diferencias significativas entre ellas, obteniéndose mayores valores de aproximación profunda en la asignatura Genética General y más superficiales en Experimientación en biotecnología. No se aprecian diferencias significativas entre niveles (grado o máster) o género, ni tampoco entre idioma de impartición en la asignatura Genética General, ni entre cursos en la asignatura Mejora genética del rendimiento en los sistemas agrarios. Para los motivos y estrategias profundas también se obtuvieron mayores valores que para los motivos y estrategias superficiales (tabla 3). Al igual que con las escalas de aproximación profunda y superficial, se observaron diferencias significativas entre asignaturas, pero no en cuestión de nivel, género, idioma o curso.

Se observaron correlaciones significativas entre los distintas escalas del cuestionario R-SPQ2F (tabla 4). Las correlaciones fueron positivas y elevadas entre DA y DS y DM y también entre SA y SM y SS, mientras que las correlaciones moderadas fueron negativas entre DA y SA, SM y SS y también entre SA y DM y DS.

Se analizó la fiabilidad interna del cuestionario utilizando el coeficiente alfa de Cronbach (tabla 5). A excepcion de la asignatura de Máster de Recursos Fitogenéticos (RF), los valores fueron superiores a 0,70 para las escala DA. Sin embargo, para las asignaturas Genetica general y Experimentación en Biotecnología se obtuvo un valor de SA inferior a 0,70., Para las escalas DM, DS, SM y SS los valores fueron, en general, inferiores a 0,70.

\section{Discusión}

Los alumnos participantes en el estudio mostraron unas puntuaciones superiores en el aprendizaje profundo. Estos valores fueron superiores (Shaik et al., 2017) o similares (Leung, Ginns \& Kember, 2008; Emilia, Bloomfield \& Rotem, 2012; Mogre \& Amalba, 2014) a los encontrados en otros estudios. En general, valores altos como estos se relacionan con una implicación mayor en los estudios que cursa el alumno, aunque se ha observado que el contexto cultural puede influir en estos valores. Por ejemplo, los estudiantes chinos suelen mostrar mayores valores de aproximación superficial (Leung, Ginns \& Kember, 2008). Valores altos como éstos se han relacionado con la intención de entender y memorizar a la vez (Leung, Ginns \& Kember, 2008). Una de las asignaturas con mayores valores de aprendizaje profundo ha sido en Genética General, de primer curso. Esto podría ser debido a la alta motivación de los alumnos al iniciar los estudios universitarios, dada la elevada nota de acceso y a que la asignatura está muy relacionada con lo que esperan del título 
universitario que van a cursar. En la asignatura Experimentación en Biotecnología se observó una mayor proporción de alumnos con un mayor valor de aprendizaje superficial con respecto al encontrado en el resto de asignaturas. Esta asignatura es de cuarto curso, totalmente práctica y de tipo optativo, por lo que los alumnos eligen cursarla y se esperaría una mayor motivación. Sin embargo, debido a las restricciones de matrícula, en muchas ocasiones los alumnos realmente no eligen la asignatura que cursan. Este hecho podría explicar que haya un mayor número de alumnos con mayor aprendizaje superficial. Además, su carácter optativo sumado a su impartición en el segundo cuatrimestre del cuarto curso, cerca del final de la titulación y coincidiendo con la elaboración del trabajo final de grado, podría justificar que los estudiantes estén más preocupados por la calificación y adopten una aproximación superficial.

Las correlaciones entre las escalas del cuestionario R-SPQ-2F fueron significativas en todos los casos, relacionándose las escalas profundas entre sí y las escalas superficiales entre sí de forma positiva, mientras que entre las escalas profundas y las escalas superficiales las correlaciones fueron negativas. Este tipo de correlaciones también se han obtenido en otros estudios (Biggs, Kember \& Leung, 2001; Leung, Ginns \& Kember, 2008) y se relacionan con percepciones del estudiantes sobre buenas estrategias de enseñanza (Fryer et al., 2012).

El análisis de fiabilidad del cuestionario utilizando el conjunto global de datos mostró valores superiores a 0,70 para DA y SA, pero no para el resto de escalas. Sin embargo, con alguna asignatura específica se obtuvieron valores menores, como es el caso de Recursos Fitogenéticos. Esto se podría explicar por el número bajo de alumnos que respondieron el cuestionario en esta asignatura. El resultado global superior a 0,70 confirmaría la fiabilidad del cuestionario, ya que este es el límite de fiababilidad propuesto para este tipo de cuestionarios (Hundleby \& Nunnally, 2006). Estos valores son similares a los de otros estudios (Immekus \& Imbrie, 2010; Socha \& Sigler, 2014; Shaik et al., 2017), pero inferiores a los obtenidos utilizando otras escalas de motivación y estrategia (Immekus \& Imbrie, 2010; Socha \& Sigler, 2014; Shaik et al., 2017). Esto ha llevado a afimar que este cuestionario solo deber ser usado para crear puntuaciones para las aproximaciones profunda y superficial, pero no para los factores de estrategia y motivación (Socha \& Sigler, 2014). Sí que se han detectado diversas asociaciones entre las preguntas y las aproximaciones de aprendizaje profunda o superficial (Biggs, Kember \& Leung, 2001; Justicia et al., 2008; Immekus \& Imbrie, 2010; Socha \& Sigler, 2014), aunque para extrapolar los resultados de esos estudios al presente caso sería necesario contar con más datos, incluyendo nuevas asignaturas de diversos cursos y titulaciones y/o cursos de evaluación. También sería necesario estudiar la relación entre los resultados académicos y las puntuaciones en el cuestionario. 
Evaluación de la aproximación al aprendizaje de estudiantes de ciencias de la vida usando la versión revisada del Study Process Questionnaire (R-SPQ-2F)

Tabla 3. Número de alumnos que respondieron el cuestionario por asignatura, tipo de asignatura y género de los alumnos (\%) y media y error estándar de las escalas de cuestionario R-SPQ-2F en la aproximación profunda (DA), aproximación superficial (SA), diferencia entre DA y SA, motivación profunda (DM), estrategia profunda (DS), motivación superficial (SM) y estrategia superficial (SS).

\begin{tabular}{|c|c|c|c|c|c|c|c|c|}
\hline & \multicolumn{2}{|c|}{$\mathrm{N}^{0}$ respuestas } & \multicolumn{2}{|l|}{ DA } & \multicolumn{2}{|l|}{$\mathbf{S A}$} & \multicolumn{2}{|c|}{ Diferencia DA-SA } \\
\hline \multicolumn{9}{|l|}{ Asignatura } \\
\hline GG & \multicolumn{2}{|l|}{$84(66,1 \%)$} & $3,29 \pm 0,06$ & $\mathrm{c}$ & $1,97 \pm 0,05$ & a & $1,32 \pm 0,08$ & $b$ \\
\hline MG & \multicolumn{2}{|l|}{$26(86,7 \%)$} & $3,17 \pm 0,11$ & $\mathrm{bc}$ & $1,90 \pm 0,10$ & $\mathrm{a}$ & $1,27 \pm 0,18$ & $\mathrm{~b}$ \\
\hline ASLB & \multicolumn{2}{|l|}{$24(24,2 \%)$} & $2,88 \pm 0,10$ & $a b$ & $2,05 \pm 0,09$ & $\mathrm{a}$ & $0,83 \pm 0,16$ & $a b$ \\
\hline $\mathrm{RF}$ & \multicolumn{2}{|l|}{$9(47,4 \%)$} & $2,94 \pm 0,13$ & $a b c$ & $2,19 \pm 0,20$ & a & $0,76 \pm 0,18$ & $\mathrm{ab}$ \\
\hline EB & \multicolumn{2}{|l|}{$39(73,6 \%)$} & $2,67 \pm 0,10$ & $\mathrm{a}$ & $2,19 \pm 0,09$ & a & $0,48 \pm 0,16$ & $\mathrm{a}$ \\
\hline \multicolumn{9}{|l|}{ Curso } \\
\hline MG 2017-18 & \multicolumn{2}{|l|}{$11(84,6 \%)$} & $3,20 \pm 0,20$ & $\mathrm{a}$ & $1,77 \pm 0,14$ & $\mathrm{a}$ & $1,43 \pm 0,28$ & $\mathrm{a}$ \\
\hline MG 2018-19 & \multicolumn{2}{|l|}{$15(88,2 \%)$} & $3,15 \pm 0,14$ & $\mathrm{a}$ & $2,00 \pm 0,13$ & $\mathrm{a}$ & $1,15 \pm 0,24$ & $\mathrm{a}$ \\
\hline \multicolumn{9}{|l|}{ Grupo } \\
\hline GG Castellano & \multicolumn{2}{|l|}{$73(71,6 \%)$} & $3,26 \pm 0,06$ & $\mathrm{a}$ & $1,98 \pm 0,05$ & $\mathrm{a}$ & $1,29 \pm 0,09$ & $\mathrm{a}$ \\
\hline GG Inglés & \multicolumn{2}{|l|}{$11(44,0 \%)$} & $3,50 \pm 0,15$ & $\mathrm{a}$ & $1,95 \pm 0,17$ & a & $1,55 \pm 0,26$ & $\mathrm{a}$ \\
\hline \multicolumn{9}{|l|}{ Tipo } \\
\hline Grado & \multicolumn{2}{|l|}{$147(55,3 \%)$} & $3,06 \pm 0,05$ & $\mathrm{a}$ & $2,04 \pm 0,04$ & $\mathrm{a}$ & $1,02 \pm 0,08$ & $\mathrm{a}$ \\
\hline Máster & \multicolumn{2}{|l|}{$35(71,4 \%)$} & $3,11 \pm 0,09$ & $\mathrm{a}$ & $1,98 \pm 0,09$ & $\mathrm{a}$ & $1,14 \pm 0,14$ & $\mathrm{a}$ \\
\hline \multicolumn{9}{|l|}{ Género } \\
\hline Femenino & \multicolumn{2}{|l|}{$127(55,5 \%)$} & $3,06 \pm 0,05$ & $\mathrm{a}$ & $1,96 \pm 0,04$ & $\mathrm{a}$ & $1,10 \pm 0,08$ & $\mathrm{a}$ \\
\hline Masculino & \multicolumn{2}{|l|}{$55(55,6 \%)$} & $3,11 \pm 0,09$ & a & $2,20 \pm 0,08$ & $\mathrm{a}$ & $0,91 \pm 0,14$ & $\mathrm{a}$ \\
\hline Total & \multicolumn{2}{|l|}{$182(55,5 \%)$} & $3,07 \pm 0,04$ & & $2,03 \pm 0,04$ & & $1,04 \pm 0,07$ & \\
\hline & \multicolumn{2}{|l|}{ DM } & \multicolumn{2}{|l|}{ DS } & SM & & SS & \\
\hline Asignatura & & & & & & & & \\
\hline GG & $3,35 \pm 0,06$ & $\mathrm{~b}$ & $3,24 \pm 0,07$ & $\mathrm{c}$ & $1,61 \pm 0,04$ & a & $2,33 \pm 0,06$ & $\mathrm{a}$ \\
\hline MG & $3,25 \pm 0,13$ & $\mathrm{~b}$ & $3,10 \pm 0,12$ & $\mathrm{bc}$ & $1,59 \pm 0,09$ & $\mathrm{a}$ & $2,22 \pm 0,14$ & $\mathrm{a}$ \\
\hline ASLB & $2,98 \pm 0,11$ & $\mathrm{ab}$ & $2,78 \pm 0,12$ & $a b$ & $1,77 \pm 0,10$ & $\mathrm{a}$ & $2,34 \pm 0,12$ & $\mathrm{a}$ \\
\hline $\mathrm{RF}$ & $3,13 \pm 0,17$ & $a b$ & $2,76 \pm 0,17$ & $a b c$ & $1,87 \pm 0,17$ & $\mathrm{a}$ & $2,51 \pm 0,24$ & $\mathrm{a}$ \\
\hline EB & $2,72 \pm 0,12$ & $\mathrm{a}$ & $2,62 \pm 0,10$ & $\mathrm{a}$ & $1,87 \pm 0,10$ & a & $2,51 \pm 0,11$ & $\mathrm{a}$ \\
\hline Curso & & & & & & & & \\
\hline MG 2017-18 & $3,13 \pm 0,06$ & $\mathrm{a}$ & $2,99 \pm 0,06$ & $\mathrm{a}$ & $1,62 \pm 0,04$ & $\mathrm{a}$ & $2,30 \pm 0,05$ & $\mathrm{a}$ \\
\hline MG 2018-19 & $3,17 \pm 0,10$ & $\mathrm{a}$ & $3,04 \pm 0,09$ & $\mathrm{a}$ & $1,88 \pm 0,08$ & a & $2,52 \pm 0,09$ & $\mathrm{a}$ \\
\hline Grupo & & & & & & & & \\
\hline GG Castellano & $3,32 \pm 0,07$ & $\mathrm{a}$ & $3,21 \pm 0,07$ & $\mathrm{a}$ & $1,61 \pm 0,05$ & $\mathrm{a}$ & $2,35 \pm 0,07$ & $\mathrm{a}$ \\
\hline GG Inglés & $3,55 \pm 0,21$ & $\mathrm{a}$ & $3,45 \pm 0,16$ & $\mathrm{a}$ & $1,67 \pm 0,17$ & a & $2,22 \pm 0,20$ & $\mathrm{a}$ \\
\hline Tipo & & & & & & & & \\
\hline Grado & $3,12 \pm 0,06$ & $\mathrm{a}$ & $3,00 \pm 0,05$ & $\mathrm{a}$ & $1,71 \pm 0,04$ & a & $2,38 \pm 0,05$ & a \\
\hline Máster & $3,22 \pm 0,10$ & $\mathrm{a}$ & $3,01 \pm 0,10$ & $\mathrm{a}$ & $1,66 \pm 0,08$ & $\mathrm{a}$ & $2,29 \pm 0,12$ & $\mathrm{a}$ \\
\hline Género & & & & & & & & \\
\hline Femenino & $3,13 \pm 0,06$ & $\mathrm{a}$ & $2,99 \pm 0,06$ & $\mathrm{a}$ & $1,62 \pm 0,04$ & $\mathrm{a}$ & $2,30 \pm 0,05$ & a \\
\hline Masculino & $3,17 \pm 0,10$ & $\mathrm{a}$ & $3,04 \pm 0,09$ & $\mathrm{a}$ & $1,88 \pm 0,08$ & $\mathrm{a}$ & $2,52 \pm 0,09$ & $\mathrm{a}$ \\
\hline Total & $3,14 \pm 0,05$ & & $3,00 \pm 0,05$ & & $1,70 \pm 0,04$ & & $2,36 \pm 0,05$ & \\
\hline
\end{tabular}

GG: Genética General; ASLB: Aspectos sociológicos y legales de la biotecnología; EB: Experimentación en biotecnología; RF: Recursos Fitogenéticos; MG: Mejora genética del rendimiento en los sistemas agrarios. Letras diferentes en la misma columna indican diferencias significativas ( $\mathrm{P}$-valor $<0,05$ ) entre grupos según el test de Tukey. 
Tabla 4. Correlación entre los distintas aproximaciones de aprendizaje de las escalas de cuestionario R-SPQ-2F de los 182 cuestionarios. Aproximación profunda (DA), aproximación superficial (SA), , motivación profunda (DM), estrategia profunda (DS), motivación superficial (SM) y estrategia superficial (SS).

\begin{tabular}{cccccc}
\hline \multicolumn{7}{c}{$\mathbf{D A}$} & $\mathbf{S A}$ & $\mathbf{D M}$ & $\mathbf{D S}$ & $\mathbf{S M}$ \\
\hline $\mathbf{S A}$ & $-0,36^{* * *}$ & & & & \\
$\mathbf{D M}$ & $0,91 * * *$ & $-0,34 * * *$ & & & \\
$\mathbf{D S}$ & $0,91^{* * *}$ & $-0,32^{* * *}$ & $0,63 * * *$ & & \\
$\mathbf{S M}$ & $-0,33^{* * *}$ & $0,85^{* * *}$ & $-0,34 * * *$ & $-0,25 * * *$ & \\
$\mathbf{S S}$ & $-0,31^{* * *}$ & $0,91 * * *$ & $-0,27 * * *$ & $-0,30 * * *$ & $0,56 * * *$ \\
\hline
\end{tabular}

$* * *: P<\overline{0,0001 . ~}$

Tabla 5. Valores del coeficiente alfa de Cronbach (Banda inferior de confianza del 95\%) entre las distintas escalas de cuestionario R-SPQ-2F de los 182 cuestionarios evaluados.

Aproximación profunda (DA), aproximación superficial (SA), motivación profunda (DM), estrategia profunda (DS), motivación superficial (SM) y estrategia superficial (SS).

\begin{tabular}{ccccccc}
\hline & DA & SA & DM & DS & SM & SS \\
\hline Asignatura & & & & & & \\
GG & $0,73(0,68)$ & $0,67(0,60)$ & $0,56(0,47)$ & $0,62(0,54)$ & $0,41(0,29)$ & $0,55(0,45)$ \\
MG & $0,79(0,75)$ & $0,72(0,67)$ & $0,69(0,63)$ & $0,63(0,56)$ & $0,59(0,51)$ & $0,62(0,54)$ \\
ASLB & $0,71(0,65)$ & $0,62(0,54)$ & $0,55(0,45)$ & $0,67(0,60)$ & $0,44(0,32)$ & $0,51(0,40)$ \\
RF & $0,31(0,18)$ & $0,77(0,72)$ & $0,11(-0,08)$ & $0,12(-0,06)$ & $0,44(0,32)$ & $0,57(0,48)$ \\
EB & $0,83(0,79)$ & $0,75(0,70)$ & $0,79(0,74)$ & $0,59(0,50)$ & $0,58(0,49)$ & $0,61(0,53)$ \\
\hline Curso & & & & & \\
MG 2017-18 & $0,87(0,85)$ & $0,75(0,70)$ & $0,82(0,79)$ & $0,70(0,64)$ & $0,67(0,60)$ & $0,67(0,60)$ \\
MG 2018-19 & $0,73(0,67)$ & $0,69(0,63)$ & $0,58(0,50)$ & $0,62(0,54)$ & $0,52(0,42)$ & $0,55(0,46)$ \\
\hline
\end{tabular}

\begin{tabular}{|c|c|c|c|c|c|c|}
\hline \multicolumn{7}{|l|}{ Grupo } \\
\hline \multicolumn{7}{|l|}{ GG } \\
\hline Castellano & $0,74(0,69)$ & $0,64(0,57)$ & $0,54(0,45)$ & $0,64(0,56)$ & $0,35(0,22)$ & $0,55(0,46)$ \\
\hline GG Inglés & $0,66(0,60)$ & $0,79(0,75)$ & $0,65(0,58)$ & $0,52(0,42)$ & $0,60(0,51)$ & $0,61(0,53)$ \\
\hline \multicolumn{7}{|l|}{ Tipo } \\
\hline Grado & $0,80(0,77)$ & $0,70(0,64)$ & $0,70(0,64)$ & $0,66(0,60)$ & $0,50(0,40)$ & $0,57(0,48)$ \\
\hline Máster & $0,73(0,68)$ & $0,74(0,69)$ & $0,58(0,50)$ & $0,56(0,47)$ & $0,57(0,48)$ & $0,60(0,52)$ \\
\hline \multicolumn{7}{|l|}{ Género } \\
\hline Femenino & $0,78(0,74)$ & $0,65(0,59)$ & $0,67(0,60)$ & $0,62(0,55)$ & $0,30(0,15)$ & $0,55(0,46)$ \\
\hline Masculino & $0,81(0,77)$ & $0,76(0,71)$ & $0,70(0,64)$ & $0,69(0,62)$ & $0,66(0,58)$ & $0,61(0,53)$ \\
\hline Total & $0,79(0,75)$ & $0,71(0,65)$ & $0,68(0,61)$ & $0,64(0,57)$ & $0,51(0,41)$ & $0,57(0,48)$ \\
\hline
\end{tabular}

La mayor aproximación profunda al aprendizaje que muestran los alumnos del presente estudio puede deberse a aspectos motivacionales. En este sentido cabe destacar que los alumnos que cursan los estudios del Grado en Biotecnología tiene una nota de admisión muy elevada (Universitat Politècnica de València, 2018), siendo la tercera en España para estos estudios (Notas de corte 2018, 2018). Esto puede conducir a que los estudiantes tengan una mayor implicación. De igual forma, los alumnos del máster en Mejora Genética Vegetal muestran un alto grado de implicación. Por otra parte, las asignaturas objeto del presente estudio están muy relacionadas con la finalidad de los estudios, por lo que la motivación del 
Evaluación de la aproximación al aprendizaje de estudiantes de ciencias de la vida usando la versión revisada del Study Process Questionnaire (R-SPQ-2F)

estudiante suele ser elevada, explicando el mayor valor de la escala de aprendizaje profundo frente al aprendizaje superficial.

\section{Conclusiones}

Dentro del ámbito de los estudios de ciencias de la vida en España parece que el cuestionario R.SPQ-2F sería fiable a la hora de valorar la aproximación al aprendizaje superficial y profunda. Así, lo indican las altas correlaciones y los índices de fiabilidad encontrados para estas escalas. Sin embargo, no sería apto para evaluar las escalas secundarias de motivación y estrategia. En este caso se obtuvieron correlaciones elevadas entre las subescalas de motivación y estrategia y la escala de aproximación correspondiente, pero no entre las propias subescalas, y además se obtuvieron valores inferiores de la alfa de Cronbach para las subescalas, especialmente las superficiales. En este caso sería necesario disponer de un mayor número de encuestas y de resultados académicos para poder analizar con mayor detalle la relación entre los ítems del cuestionario y las subescalas.

En general, en el contexto considerado, los estudiantes, manifestaron mayoritariamente una aproximación alta al aprendizaje profundo, lo que podría deberse a una mayor implicación en los estudios. En este sentido, se observaron diferencias entre asignaturas en los valores obtenidos para aprendizaje profundo, pero no para aprendizaje superficial. Sin embargo, estas diferencias no se observaron al analizar el género de los estudiantes, nivel de estudio, ni para el idioma de impartición de las clases o el curso escolar en los casos en que se tuvo en cuenta.

Los resultados obtenidos implican un buen punto de partida de los estudiantes en los estudios de ciencias de la vida, ya que su aproximación al aprendizaje es la adecuada. Sería necesario continuar el estudio para valorar con mayor precision las subescalas y profundizar en el papel de la motivación para explicar las diferencias encontradas entre asignaturas.

\section{Agradecimientos}

La publicación de este trabajo ha sido parcialmente financiada por un proyecto de innovación educativa (PIME/2017/A/016/A) concedido por el Vicerrectorado de Estudios, Calidad y Acreditación de la Universitat Politècncia de València (UPV).

\section{Referencias}

Berger, J. L. \& KARABENICK, S. A. (2011) 'Motivation and students' use of learning strategies: Evidence of unidirectional effects in mathematics classrooms', Learning and Instruction. Elsevier Ltd, 21(3), pp. 416-428. doi: 10.1016/j.learninstruc.2010.06.002.

Biggs, J. (1987) Student Approaches to Learning and Studying. Research Monograph., Australian Education Research and Development. Available at: https://eric.ed.gov/?id=ED308201.

Biggs, J. (1999) 'What the Student Does: teaching for enhanced learning', Higher Education Research \& Development, 18(1), pp. 57-75. doi: 10.1080/0729436990180105. 
BigGS, J. B. (1978) 'Individual and group differences in study processes', British Journal of Educational Psychology, 48(3), pp. 266-279. doi: 10.1111/j.2044-8279.1978.tb03013.x.

Biggs, J., KemBer, D. \& LeUnG, D. Y. P. (2001) ‘The revised two-factor Study Process Questionnaire: R-SPQ-2F’, British Journal of Educational Psychology, 71(1), pp. 133-149. doi: 10.1348/000709901158433.

Boyle, E. A., Duffy, T. \& DunLeAvy, K. (2003) 'Learning styles and academic outcome: The validity and utility of Vermunt's Inventory of Learning Styles in a British higher education setting', British Journal of Educational Psychology, 73(2), pp. 267-290. doi: 10.1348/00070990360626976.

Chamorro-Premuzic, T., Furnham, A. \& Lewis, M. (2007) 'Personality and approaches to learning predict preference for different teaching methods', Learning and Individual Differences, 17(3), pp. 241-250. doi: 10.1016/j.lindif.2006.12.001.

CRAWFORD, K. ET AL. (1998) 'Qualitatively different experiences of learning mathematics at university', Learning and Instruction, 8(5), pp. 455-468. doi: 10.1016/S0959-4752(98)00005-X.

Emilia, O., BloOmfield, L. \& Rotem, A. (2012) 'Measuring students' approaches to learning in different clinical rotations', BMC Medical Education, 12(1), p. 114. doi: 10.1186/1472-6920-12-114.

ENTWiSTLE, N. J. (1991) 'Approaches to learning and perceptions of the learning environment', Higher Education, 22(3), pp. 201-204. doi: 10.1007/BF00132287.

ENTWISTLE, N. J. \& TAIT, H. (1990) 'Approaches to learning, evalutions of teaching, and perferences for contrasting academic environments’, Higher Eduation, 19(2), pp. 291-299.

EnTwistLe, N. J. \& TAIT, H. (1995) The revised approaches to studying inventory. Edinburgh, Scotland: Centre for Research on Learning and Instruction.

Fox, R. A., McManus, I. C. \& Winder, B. C. (2001) 'The shortened Study Process Questionnaire: An investigation of its structure and longitudinal stability using confirmatory factor analysis', British Journal of Educational Psychology, 71(4), pp. 511-530. doi: 10.1348/000709901158659.

FrĂSINEANU, E. S. (2013) 'Approach to Learning Process: Superficial Learning and Deep Learning at Students', Procedia - Social and Behavioral Sciences, 76, pp. 346-350. doi: 10.1016/j.sbspro.2013.04.125.

FRYER, L. K. ET AL. (2012) 'The adaptation and validation of the CEQ and the R-SPQ-2F to the Japanese tertiary environment', British Journal of Educational Psychology, 82(4), pp. 549-563. doi: 10.1111/j.20448279.2011.02045.x.

Furnham, A., Monsen, J. \& Ahmetoglu, G. (2009) 'Typical intellectual engagement, Big Five personality traits, approaches to learning and cognitive ability predictors of academic performance', British Journal of Educational Psychology, 79(4), pp. 769-782. doi: 10.1348/978185409X412147.

GiJBELS, D. ET AL. (2005) 'The relationship between students' approaches to learning and the assessment of learning outcomes', European Journal of Psychology of Education, 20(4), pp. 327-341. doi: 10.1007/BF03173560.

Gordon, C. \& DeBus, R. (2002) 'Developing deep learning approaches and personal teaching efficacy within a preservice teacher education context', British Journal of Educational Psychology, 72(4), pp. 483-511. doi: $10.1348 / 00070990260377488$

Gow, L. \& KemBER, D. (1990) 'Does higher education promote independent learning?', Higher Education, 19(3), pp. 307-322. doi: 10.1007/BF00133895.

GRABINGER, R. S. \& DUNLAP, J. C. (1995) 'Rich environments for active learning: a definition', ALT-J, 3(2), pp. 534. doi: $10.1080 / 0968776950030202$.

Graham, N. C., Entwistle, N. \& Ramsden, P. (1984) 'Understanding Student Learning', British Journal of Educational Studies, 32(3), p. 284. doi: 10.2307/3121589. 
Evaluación de la aproximación al aprendizaje de estudiantes de ciencias de la vida usando la versión revisada del Study Process Questionnaire (R-SPQ-2F)

HARPER, G. \& KemBer, D. (1986) 'Approaches to Study of Distance Education Students', British Journal of Educational Technology, 17(3), pp. 212-222. doi: 10.1111/j.1467-8535.1986.tb00510.x.

Hundleby, J. D. \& Nunnally, J. (2006) Psychometric Theory, American Educational Research Journal. Edited by McGraw-Hil. New York, USA. doi: 10.2307/1161962.

Immekus, J. C. \& IMBrie, P. K. (2010) 'A Test and Cross-Validation of the Revised Two-Factor Study Process Questionnaire Factor Structure Among Western University Students’, Educational and Psychological Measurement, 70(3), pp. 495-510. doi: 10.1177/0013164409355685.

Justicia, F. ET AL. (2008) 'The Revised Two-Factor Study Process Questionnaire (R-SPQ-2F): Exploratory and confirmatory factor analyses at item level', European Journal of Psychology of Education, 23(3), pp. 355-372. doi: 10.1007/BF03173004.

Leung, D. Y. P., GinNS, P. \& KeMBER, D. (2008) 'Examining the Cultural Specificity of Approaches To Learning in Universities in Hong Kong and Sydney’, Journal of Cross-Cultural Psychology, 39(3), pp. 251-266. doi: $10.1177 / 0022022107313905$.

LEUNG, S. F., MOK, E. \& WONG, D. (2008) 'The impact of assessment methods on the learning of nursing students', Nurse Education Today, 28(6), pp. 711-719. doi: 10.1016/j.nedt.2007.11.004.

MARTON, F. (1981) 'Phenomenography? Describing conceptions of the world around us', Instructional Science, 10(2), pp. 177-200. doi: 10.1007/BF00132516.

MARTON, F. \& SÄLJÖ, R. (1976a) 'On qualitative differences in learning-II outcome as a function of the learners's conception of the task', British Journal of Educational Psychology, 46(2), pp. 115-127. doi: 10.1111/j.20448279.1976.tb02304.x.

MARTON, F. \& SÄLJÖ, R. (1976b) 'On qualitative differences in learning: I-Outcome and process', British Journal of Educational Psychology, 46(1), pp. 4-11. doi: 10.1111/j.2044-8279.1976.tb02980.x.

MAY, W. ET AL. (2012) 'The relationship between medical students' learning approaches and performance on a summative high-stakes clinical performance examination', Medical Teacher, 34(4), pp. e236-e241. doi: $10.3109 / 0142159$ X.2012.652995.

Michael, W. B., Michael, J. J. \& Zimmerman, W. S. (1985) Study Attitudes and Methods Survey (SAMS). San Diego, CA: Educational and Industrial Testing Service.

Mogre, V. \& Amalba, A. (2014) 'Assessing the reliability and validity of the Revised Two Factor Study Process Questionnaire (RSPQ2F) in Ghanaian medical students', Journal of Educational Evaluation for Health Professions, 11, p. 19. doi: 10.3352/jeehp.2014.11.19.

Muñoz San Roque, I., Prieto Navarro, L. \& Torre Puente, J. C. (2012) 'Enfoques de aprendizaje, autorregulación, autoeficacia, competencias y evaluación. Un estudio descriptivo de estudiantes de educación infantil y primaria', in Torre Puente, J. C. (ed.) Educación y nuevas sociedades. Madrid: Universidad Pontificia Comillas, pp. 237-266.

Notas de corte 2018 (2018) El País. Available at: https://elpais.com/especiales/universidades/titulacion/notas/biotecnologia/36 (Accessed: 24 September 2018).

PHAN, H. P. (2008) 'Multiple regression analysis of epistemological beliefs, learning approaches and self-regulated learning', Electronic Journal of Research in Educational Psychology, N.14, Vol 6 (1) 2008, 157-184, 6(1), pp. 157184.

Prosser, M. \& TRigwell, K. (1999) Understanding learning and leaching. The experience in higher education. Buckingham: Open University Press.

QS ENRolment Solutions (2017) International Student Survey 2017. Available at:

(cc)) BY-NC-ND 2019, Universitat Politècnica de València 
https://www.internationalstudentsurvey.com/international-student-survey-2017/ (Accessed: 4 May 2018).

RichaRdson, J. T. E. \& KING, E. (1998) ‘Adult Students in Higher Education: Burden or Boon?', The Journal of Higher Education, 69(1), p. 65. doi: 10.2307/2649182.

Rossum, E. J. \& SCHENK, S. M. (1984) 'The relationship between learning conceptions, study strategy and learning outcome', British Journal of Educational Psychology, 54(1), pp. 73-83. doi: 10.1111/j.2044-8279.1984.tb00846.x.

SALAMONSON, Y. ET AL. (2013) 'Learning approaches as predictors of academic performance in first year health and science students', Nurse Education Today. Elsevier Ltd, 33(7), pp. 729-733. doi: 10.1016/j.nedt.2013.01.013.

SchmeCK, R. R., RiBich, F. \& RAMANAIAH, N. (1977) 'Development of a Self-Report Inventory for Assessing Individual Differences in Learning Processes', Applied Psychological Measurement, 1(3), pp. 413-431. doi: $10.1177 / 014662167700100310$.

SEVERIENS, S. \& DAM, G. (1998) ‘A multilevel meta-analysis of gender differences in learning orientations', British Journal of Educational Psychology, 68(4), pp. 595-608. doi: 10.1111/j.2044-8279.1998.tb01315.x.

SHAIK, S. A. ET AL. (2017) 'Assessing Saudi medical students learning approach using the revised two-factor study process questionnaire', International Journal of Medical Education, 8, pp. 292-296. doi: 10.5116/ijme.5974.7a06.

SHuELl, T. J. (1986) ‘Cognitive Conceptions of Learning', Review of Educational Research, 56(4), pp. 411-436. doi: $10.3102 / 00346543056004411$.

Skogsberg, K. \& Clump, M. (2003) 'Do psychology and biology majors differ in their study processes and learning styles?', College Student Journal, 37(1), pp. 27-33.

SNelgrove, S. \& Slater, J. (2003) 'Approaches to learning: psychometric testing of a study process questionnaire’, Journal of Advanced Nursing, 43(5), pp. 496-505. doi: 10.1046/j.1365-2648.2003.02747.x.

SochA, A. \& SigleR, E. A. (2014) 'Exploring and "reconciling" the factor structure for the Revised Two-factor Study Process Questionnaire', Learning and Individual Differences. Elsevier Inc., 31, pp. 43-50. doi: 10.1016/j.lindif.2013.12.010.

Stes, A., De Maeyer, S. \& Van Petegem, P. (2013) 'Examining the Cross-Cultural Sensitivity of the Revised Two-Factor Study Process Questionnaire (R-SPQ-2F) and Validation of a Dutch Version', PLoS ONE. Edited by O. García, 8(1), p. e54099. doi: 10.1371/journal.pone.0054099.

Trigwell, K., Prosser, M. \& Waterhouse, F. (1999) 'Relations between teachers' approaches to teaching and students' approaches to learning', Higher Education, 37(1), pp. 57-70. doi: 10.1023/A:1003548313194.

Universitat Politècnica de VAlÈncia (2018) Notas de corte curso 2018-19. Available at: http://www.upv.es/pls/oalu/sic_futuroalumno.notascorte?p_idioma=c (Accessed: 24 September 2018).

VERMUNT, J. D. (1994) Inventory of Learning Styles in Higher Education: Scoring key. Tilburg, The Netherlands: Tilburg University, Department of Educational Psychology.

WeINSTEIN, C. E. (1987) Learning and Study Strategies Inventory. Clearwater, FL: H \& H Publishing.

Wilson, K. L., SMART, R. M. \& WATSON, R. J. (1996) 'Gender differences in approaches to learning in first year psychology students', British Journal of Educational Psychology, 66(1), pp. 59-71. doi: 10.1111/j.20448279.1996.tb01176.x.

ZEEGERS, P. (2001) 'Approaches to learning in science: A longitudinal study', British Journal of Educational Psychology, 71(1), pp. 115-132. doi: 10.1348/000709901158424.

Zimmerman, B. J. (1986) 'Becoming a self-regulated learner: Which are the key subprocesses?', Contemporary Educational Psychology, 11(4), pp. 307-313. doi: 10.1016/0361-476X(86)90027-5. 